J. Lake Sci. (湖泊科学) , 2013, 25(1): 16-22

http: //www.jlakes.org. E-mail : jlakes@niglas. ac.cn

(c) 2013 by Journal of Lake Sciences

\title{
改性粘土辅助沉水植物修复技术维持清水稳态的原位研究”
}

\author{
汤 至金 ${ }^{1}$ 特 $^{2}$, 倪乐意 $^{2}$, 谢 平 ${ }^{1,2 * *}$ \\ (1:华中农业大学水产学院,武汉 430070) \\ (2: 中国科学院水生生物研究所东湖台站,武汉 430072)
}

\begin{abstract}
摘 要: 在富营养湖泊治理实践中, 修复沉水植被被认为是改善水质的长效措施, 而壳聚糖改性粘土是短期快速改善水 质的有效手段. 本研究利用改性粘土辅助沉水植被修复,旨在探索改善水质的长效方案. 2011 年 5-11 月在太湖梅梁湾 开展了四组不同处理 (对照、水草、水草 + 粘土、粘土) 围隔实验, 在水草 (盖度 $13.0 \%$ ) 和水草 + 粘土 (盖度 $52.3 \%$ ) 围隔 中不同程度重建了苦草群落. 实验期内每 $3 \mathrm{~d}$ 一次的水质监测表明, 粘土处理可显著改善水质, 水体总氮 (TN)、总磷 ( TP) 、正磷酸盐 $\left(\mathrm{PO}_{4}^{3-}-\mathrm{P}\right)$ 和叶绿素 $\mathrm{a}(\mathrm{Chl} . \mathrm{a})$ 含量分别比对照下降了 $20.7 \% 、 74.6 \% 、 31.0 \%$ 和 $80.4 \%$, 透明度 $(\mathrm{SD})$ 升高 了 $90.4 \%$; 粘土辅助植被修复改善水质效果最长稳, 水体 $\mathrm{TN} 、 \mathrm{TP} 、 \mathrm{PO}_{4}^{3-}-\mathrm{P}$ 和 Chl. a 含量分别比对照下降了 $36.2 \%$ 、 $64.0 \% 、 28.6 \%$ 和 $71.1 \%, \mathrm{SD}$ 升高了 $76.4 \%$; 低盖度苦草群落单独处理对水质改善效果不显著. 在三种处理中, 粘土辅助植 被修复改善底质效果最好, 使间隙水的 $\mathrm{TN} 、 \mathrm{TP} 、 \mathrm{PO}_{4}^{3-}-\mathrm{P} 、 \mathrm{NH}_{4}^{+}-\mathrm{N}$ 分别比实验前下降了 $15.6 \% 、 61.7 \% 、 55.8 \%$ 和 $82.8 \%$. 本研 究表明改性粘土辅助沉水植被修复可作为重富营养水体中水质改善的整合技术, 但其长期生态效应仍需谨慎评估.
\end{abstract}

关键词: 富营养化水体; 苦草; 壳聚糖改性粘土;沉水植被修复;水质改善

\section{In situ study on the maintenance of clear water by restoration of submersed macrophytes with the aid of modified soils}

\author{
TANG Xin ${ }^{1}$, CAO Te ${ }^{2}$, NI Leyi ${ }^{2} \&$ XIE Ping ${ }^{1,2}$ \\ (1: College of Fisheries, Huazhong Agricultural University, Wuhan 430070, P. R. China) \\ (2: Donghu Experimental Station of Lake Ecosystems, Institute of Hydrobiology, Chinese Academy of Sciences, Wuhan \\ 430072, P. R. China)
}

\begin{abstract}
Restoration of submersed vegetation is generally considered as an effective long-term approach to improve the water quality of eutrophic lakes, and chitosan-modified soil has been used to rapidly improve the water quality in the recent years. In this study, an experiment with four treatments ( the control, submersed macrophyte, submersed marcophyte + soil, and soil) was carried out in the hypereutrophic water(Meiliang Bay) in Lake Taihu during May to December in 2011, with the aim to develop a macrophyte-mediated approach to improve water quality. During the period of the experiment, submersed vegetation had a final coverage of $13.0 \%$ and $52.3 \%$ in the submersed macrophyte and the submersed macrophyte + soil treatments, respectively. The indices of water quality were measured in 3-day intervals. The results showed that the soil was effective to improve the water quality, with a decrease in the contents of $\mathrm{TP}$ by $74.6 \%$, TN by $20.7 \%, \mathrm{PO}_{4}^{3-}-\mathrm{P}$ by $31.0 \%$, Chl. a by $80.4 \%$ and an increased $\mathrm{SD}$ by $90.4 \%$ as compared to the control; the submersed marcophyte + soil treatment was the most effective way to decrease the contents of TP (64.0\%), $\mathrm{TN}(36.2 \%), \mathrm{PO}_{4}^{3-}-\mathrm{P}(28.6 \%)$ and $\mathrm{Chl} . \mathrm{a}(71.1 \%)$, and increased the $\mathrm{SD}(76.4 \%)$. The submersed marcophyte restoration alone did not improve the water quality. The submersed marcophyte + soil treatment was also the most effective one among the three treatments to decrease the contents of $\mathrm{TN}(15.6 \%), \mathrm{TP}(61.7 \%), \mathrm{PO}_{4}^{3-}-\mathrm{P}(55.8 \%)$ and $\mathrm{NH}_{4}^{+}-\mathrm{N}(82.8 \%)$ in the sediment interstitial water. The study implies that restoration of submersed vegetation with the aid of chitosan-modified soil be an effective technology to improve the water quality, although the effectiveness needs to be further evaluated on a long-term basis.
\end{abstract}

* 国家重点基础研究发展计划 “973” 项目 (2008CB418105) 和国家水体污染控制与治理科技重大专项项目 (2009ZX07101-013) 联合资助. 2012-03-19 收稿; 2012-08-10 收修改稿. 汤金畋, 男, 1986 年生, 硕士研究生; E-mail:tangxin600@163.com.

** 通信作者;E-mail : xieping@ ihb. ac. cn. 
Keywords: Eutrophic water; Vallisneria natans; chitosan-modified soils; submersed macrophyte restoration; water quality improvement

当前, 富营养化水体中的蓝藻水华和水生植被衰退是全世界面临的水污染问题 ${ }^{[-2]}$. 水生植被, 尤其是 沉水植被, 作为湖泊生态系统的重要初级生产者, 可以降低湖泊水体营养盐负荷、控制藻类生长、保持水体 的清水稳态和较高的生物多样性, 其生态重建或修复被认为是湖泊生态修复的重要举措之一 ${ }^{[3-5]}$. 国内外利 用水生植物治理水体富营养化的研究很多 ${ }^{[6-8]}$, 在沉水植被修复过程中因受到水下光照条件差、氮磷营养盐 过高、底质松软、水体溶解氧供给不足等环境条件的制约,沉水植被在这些水体中很难存活. 此外,在选择被 恢复沉水植物物种上, 其耐污能力、适宜的移栽期和营养条件、有效的繁殖和移植技术都会影响植被修复的 效果. 水鳖科苦草 (Vallisneria natans) 是我国长江中下游区域常见的多年生沉水植物, 耐污和适应性较强, 常 在沉水植被修复中使用.

Anderson ${ }^{[9]}$ 较早就提出了粘土絮凝除藻是很有前景的方法, 随后研究者发现利用粘土作为载体并通过 不同物质对其进行改性,已成为这一研究领域的新动向. 邹华和潘纲等 ${ }^{[10-11]}$ 研究了使用壳聚糖改性粘土对 微囊藻水华的去除效应, 并在太湖梅梁湾水域进行了围隔实验, 结果表明投加少量的壳聚糖改性粘土后不 仅有效地去除了藻华, 且水体透明度得到较大改善, 营养盐含量也有显著降低. 他们还比较了 26 种粘土除 藻效率和效果, 提出了壳聚糖改性粘土除藻的网捕和架桥机理,并提出采用当地粘土原位去除蓝藻水华是 可行的 ${ }^{[12-13]}$. 改性粘土有来源充足、天然无毒、使用方便、节约资源等特点, 其絮凝作用作为一种新兴的应急 除藻技术, 越来越多地在水质改善中使用.

本研究采用沉水植被重建与改性粘土处理相结合的方法,利用沉水植被改善水质的长效性与稳定性及 壳聚糖改性粘土改善水质的短期高效性, 通过改性粘土絮凝作用短期改善生境条件,创造沉水植物移栽条 件, 使沉水植物易于存活生长, 并产生长期稳定水质功能. 本实验通过监测不同种类处理围隔中水质和底泥 间隙水的营养盐含量变化和苦草的盖度, 研究改性粘土修复沉水植被及对蓝藻水华的抑制效果, 旨在探索 富营养水体水质改善的长效措施.

\section{1 材料与方法}

\section{1 实验地点与材料}

原位围隔实验于 2011 年 5-11 月在太湖北部的梅梁湾进行, 其湖水深度为 $0.8 \sim 1.8 \mathrm{~m}$, 大部分水域无 大型水生植物分布, 是典型的藻型富营养化湖区 ${ }^{[14]}$. 实验系统由 12 个 PVC 透地围隔 $(2.5 \mathrm{~m} \times 2.5 \mathrm{~m})$ 构成, 围隔布的底边采用石龙埋藏以防止围隔内外水体交换, 用钢管固定于水体中. 实验开始前所有围隔布均沉 于湖水中静置两星期, 待围隔内外水体充分平衡. 实验采取了 4 种处理措施: 对照 (不做处理)、加改性粘土 (粘土)、移栽苦草并添加改性粘土 (水草 + 粘土)、只移栽苦草 (水草). 每种处理采用 3 个围隔重复实验, 实 验围隔附近的湖水作为本底参考, 在实验点附近采用竹排消浪.

实验苦草种苗取自太湖, 株高 $35 \sim 40 \mathrm{~cm}$, 大小均匀, 叶片颜色鲜亮, 无明显损伤. 在水草 + 粘土处理和 水草处理共 6 个围隔中, 采用人工扞插方式移栽苦草, 苦草种苗密度为 600 株/围隔. 改性粘土处理参考 Pan 等 ${ }^{[12-13]}$ 的方法, 改性剂为壳聚糖, 使用前配置成 $1 \mathrm{mg} / \mathrm{ml}$ 的壳聚糖盐酸溶液 ${ }^{[15]}$. 粘土购自江苏玖川纳米材料 科技有限公司生产的 JC-ZF-01 型水处理剂, 主要成分为高岭石. 壳聚糖与粘土的含量按 1:100 的质量比例 计算. 改性粘土用量按围隔水体重量的 $0.3 \% 0 \sim 1.0 \%$ 计算. 改性粘土具体使用方法为经加人适量围隔水摚 拌形成悬浊液,均匀泼酒于实验处理围隔,改性粘土泼酒频率为 $6 \mathrm{~d} /$ 次, 实验期间共泼酒 10 次.

\section{2 实验样品采集与分析}

实验样品采集期为 5 月 30 日至 7 月 28 日,共 $60 \mathrm{~d}$. 围隔内水样每 $3 \mathrm{~d}$ 采集一次, 用于测定水体的总氮 $(\mathrm{TN})$ 、总磷 $(\mathrm{TP})$ 、硝态氮 $\left(\mathrm{NO}_{3}^{-}-\mathrm{N}\right)$ 、铵态氮 $\left(\mathrm{NH}_{4}^{+}-\mathrm{N}\right)$ 、正磷酸盐 $\left(\mathrm{PO}_{4}^{3-}-\mathrm{P}\right)$ 和叶绿素 $\mathrm{a}(\mathrm{Chl} . \mathrm{a})$. 测定方法参 考文献 [16-17]. 水体透明度 $(\mathrm{SD})$ 用透明度盘测定. 各围隔在实验开始前和结束后采集表层沉积物,离心后 上覆水用于测定底泥间隙水体的 $\mathrm{TN} 、 \mathrm{TP} 、 \mathrm{NH}_{4}^{+}-\mathrm{N} 、 \mathrm{PO}_{4}^{3-}-\mathrm{P} 、 \mathrm{NO}_{3}^{-}-\mathrm{N}$. 实验结束后采集 $0.25 \mathrm{~m}^{2}$ 苦草, 计算苦草 密度并推算围隔内苦草的盖度. 


\section{3 数据统计分析}

图 $1 、 2 、 3$ 中的值表示形式为平均值 \pm 标准差. 所有数据的方差分析、Tukey HSD 多重比较检验、 $t$ 检验由 软件 Statistica 6.0 完成, $P<0.05$ 时统计上认为是显著的.

\section{2 结果}

\section{1 不同处理下围隔水体水质变化}

添加改性粘土和移栽苦草对围隔内水质影响显著 (表 $1, P<0.05$ ), 围隔效应也不同程度地影响水质 (图 1). 实验期间, 太湖水体 TP 含量为 $0.10 \mathrm{mg} / \mathrm{L}$, 对照围隔水体 TP 含量为 $0.13 \mathrm{mg} / \mathrm{L}$, 移栽水草围隔 TP 含 量为 $0.12 \mathrm{mg} / \mathrm{L}$, 粘土围隔 TP 含量为 $0.04 \mathrm{mg} / \mathrm{L}$, 水草 + 粘土围隔 TP 含量为 $0.05 \mathrm{mg} / \mathrm{L}$, 添加粘土使 TP 下降 约 $60 \%$, 仅移栽苦草对 TP 影响不显著 (图 1A), 但两者有较显著交互作用 (表 $1, P<0.05$ ); 太湖水体 $\mathrm{PO}_{4}^{3-}-\mathrm{P}$ 含量为 $0.013 \mathrm{mg} / \mathrm{L}$, 对照和水草围隔水体的 $\mathrm{PO}_{4}^{3-}-\mathrm{P}$ 为 $0.008 \mathrm{mg} / \mathrm{L}$, 粘土和水草 + 粘土围隔 $\mathrm{PO}_{4}^{3-}-\mathrm{P}$ 约为 $0.006 \mathrm{mg} / \mathrm{L}$, 添加粘土使 $\mathrm{PO}_{4}^{3-}-\mathrm{P}$ 下降约 30\%, 水草处理对 $\mathrm{PO}_{4}^{3-}-\mathrm{P}$ 影响不显著(图 1C).

表 1 不同处理围隔内水体和间隙水的水质指标的方差检验 1 )

Tab. 1 ANOVA of the water and interstitial water quality indices in the different enclosure treatments

\begin{tabular}{|c|c|c|c|c|c|c|c|c|c|c|c|c|}
\hline \multirow{2}{*}{ 方差来源 } & \multicolumn{2}{|c|}{$\mathrm{TP}$} & \multicolumn{2}{|c|}{$\mathrm{TN}$} & \multicolumn{2}{|c|}{$\mathrm{PO}_{4}^{3-}-\mathrm{P}$} & \multicolumn{2}{|c|}{$\mathrm{NH}_{4}^{+}-\mathrm{N}$} & \multicolumn{2}{|c|}{$\mathrm{NO}_{3}^{-}-\mathrm{N}$} & \multirow{2}{*}{$\begin{array}{c}\text { Chl. a } \\
\text { 水体 }\end{array}$} & \multirow{2}{*}{$\begin{array}{c}\mathrm{SD} \\
\text { 水体 }\end{array}$} \\
\hline & 水体 & 间隙水 & 水体 & 间隙水 & 水体 & 间隙水 & 水体 & 间隙水 & 水体 & 间隙水 & & \\
\hline 粘土 & $0.466 * *$ & $0.364^{* *}$ & $0.144^{* *}$ & 0.000 & $0.071^{* *}$ & $0.302^{* *}$ & $0.095^{* *}$ & 0.000 & $0.122^{* *}$ & $0.159^{* *}$ & $0.489^{* *}$ & $0.339^{* *}$ \\
\hline 水草 & 0.000 & $0.200^{* *}$ & $0.029^{* *}$ & $0.353^{* *}$ & 0.000 & $0.239^{* *}$ & $0.023 * *$ & 0.001 & $0.021 * *$ & 0.016 & 0.000 & 0.000 \\
\hline 时间 & $0.212 * *$ & 0.012 & $0.482 * *$ & $0.131 * *$ & $0.606^{* *}$ & 0.031 & $0.422 * *$ & $0.859^{* *}$ & $0.478^{* *}$ & 0.074 & $0.120^{* *}$ & $0.348^{* *}$ \\
\hline 水草 $\times$ 粘土 & $0.008 * *$ & 0.034 & 0.004 & 0.003 & 0.002 & $0.042^{* *}$ & 0.019 ** & 0.003 & $0.048^{* *}$ & $0.101^{* *}$ & $0.008^{* *}$ & $0.004^{* *}$ \\
\hline 粘土 $\times$ 时间 & $0.129^{* *}$ & $0.204^{* *}$ & $0.089^{* *}$ & 0.037 & $0.077^{* *}$ & $0.229^{* *}$ & $0.112 * *$ & 0.000 & $0.088^{* *}$ & 0.019 & $0.229^{* *}$ & $0.185^{* *}$ \\
\hline
\end{tabular}

1) 表中数据为对应因素离差平方和与总离差平方和的比值, $* *$ 表示差异显著 $(P<0.05)$.

在氮含量上, 太湖水体和对照围隔的 TN 含量约为 $1.5 \mathrm{mg} / \mathrm{L}$, 移栽水草围隔 TN 含量为 $1.4 \mathrm{mg} / \mathrm{L}$, 粘土 围隔 TN 含量为 $1.2 \mathrm{mg} / \mathrm{L}$, 水草 + 粘土围隔 TN 含量为 $1.0 \mathrm{mg} / \mathrm{L}$, 添加粘土使 $\mathrm{TN}$ 含量下降约 $20 \%$, 仅移栽 苦草对 TN 影响不显著 (图 1B) ; 对照和水草围隔 $\mathrm{NH}_{4}^{+}-\mathrm{N}$ 含量约为 $0.035 \mathrm{mg} / \mathrm{L}$, 粘土围隔和太湖水体 $\mathrm{NH}_{4}^{+}-\mathrm{N}$ 含量为 $0.085 \mathrm{mg} / \mathrm{L}$, 相比对照升高约 1.5 倍, 水草 + 粘土围隔 $\mathrm{NH}_{4}^{+}-\mathrm{N}$ 含量为 $0.050 \mathrm{mg} / \mathrm{L}$, 相比对照升高约 $40 \%$, 仅移栽苦草对 $\mathrm{NH}_{4}^{+}-\mathrm{N}$ 影响不显著 (图 1D), 两者有较显著交互作用 (表 $1, P<0.05$ ); 太湖水体 $\mathrm{NO}_{3}^{-}-\mathrm{N}$ 含量为 $0.9 \mathrm{mg} / \mathrm{L}$, 对照和水草围隔 $\mathrm{NO}_{3}^{-}-\mathrm{N}$ 含量为 $0.2 \mathrm{mg} / \mathrm{L}$, 粘土围隔 $\mathrm{NO}_{3}^{-}-\mathrm{N}$ 含量为 $0.38 \mathrm{mg} / \mathrm{L}$, 添加粘土 使 $\mathrm{NO}_{3}^{-}-\mathrm{N}$ 含量相比对照上升 $90 \%$, 水草 + 粘土围隔 $\mathrm{NO}_{3}^{-}-\mathrm{N}$ 含量为 $0.23 \mathrm{mg} / \mathrm{L}$, 相比对照升高约 $15 \%$, 仅移 栽苦草对 $\mathrm{NO}_{3}^{-}-\mathrm{N}$ 影响不显著 (图 1E), 两者有显著交互作用 $($ 表 $1, P<0.05$ ).

太湖水体 Chl. a 含量为 $195 \mu \mathrm{g} / \mathrm{L}$, 对照和水草围隔水体的 Chl. a 含量约为 $50 \mu \mathrm{g} / \mathrm{L}$, 粘土和水草 + 粘土 围隔 Chl. a 含量约为 $15 \mu \mathrm{g} / \mathrm{L}$, 添加粘土使 Chl. a 含量下降约 70\%, 仅移栽苦草对 $\mathrm{Chl}$. a 影响不显著 (图 $1 \mathrm{~F}$ ), 两者交互作用较显著 (表 $1, P<0.05$ ). 太湖水体 $\mathrm{SD}$ 为 $55 \mathrm{~cm}$, 对照和水草围隔水体的 $\mathrm{SD}$ 约为 $70 \mathrm{~cm}$, 粘土和水草 + 粘土围隔 $\mathrm{SD}$ 约为 $120 \mathrm{~cm}$, 添加粘土使 $\mathrm{SD}$ 增加了 $50 \mathrm{~cm}$ 以上, 仅移栽苦草对 $\mathrm{SD}$ 影响不显著 (图 $1 \mathrm{G}$ ), 两者交互作用较显著 (表 $1, P<0.05$ ).

综上所述,水草 + 粘土处理使围隔水体 $\mathrm{TN} 、 \mathrm{TP} 、 \mathrm{PO}_{4}^{3-}-\mathrm{P}$ 和 Chl. a 含量相比对照分别降低了 $36.2 \%$ 、 $64.0 \% 、 28.6 \%$ 和 $71.1 \%, \mathrm{NH}_{4}^{+}-\mathrm{N} 、 \mathrm{NO}_{3}^{-}-\mathrm{N}$ 和 $\mathrm{SD}$ 值升高了 $38.2 \% 、 9.1 \%$ 和 $76.4 \%$. 粘土处理使围隔水体 $\mathrm{TN} 、 \mathrm{TP} 、 \mathrm{PO}_{4}^{3-}-\mathrm{P}$ 和 Chl. a 含量相比对照降低了 20.7\%、74.6\%、31.0\% 和 80.4\%, $\mathrm{NH}_{4}^{+}-\mathrm{N} 、 \mathrm{NO}_{3}^{-}-\mathrm{N}$ 和 $\mathrm{SD}$ 值升 高了 $147.3 \% 、 83.2 \%$ 和 $90.4 \%$ (图 1).

\section{2 不同处理下围隔沉积物间隙水水质变化}

不同处理下围隔底泥间隙水水质指标方差分析结果显示, 添加改性粘土和移栽苦草对 $\mathrm{TP} 、 \mathrm{PO}_{4}^{3-}-\mathrm{P}$ 和 $\mathrm{NO}_{3}^{-}-\mathrm{N}$ 影响显著 (表 $1, P<0.05$ ). 实验前对照和粘土围隔底泥间隙水 $\mathrm{TN}$ 为 $18 \mathrm{mg} / \mathrm{L}$, 实验后降到 $16 \mathrm{mg} / \mathrm{L}$; 

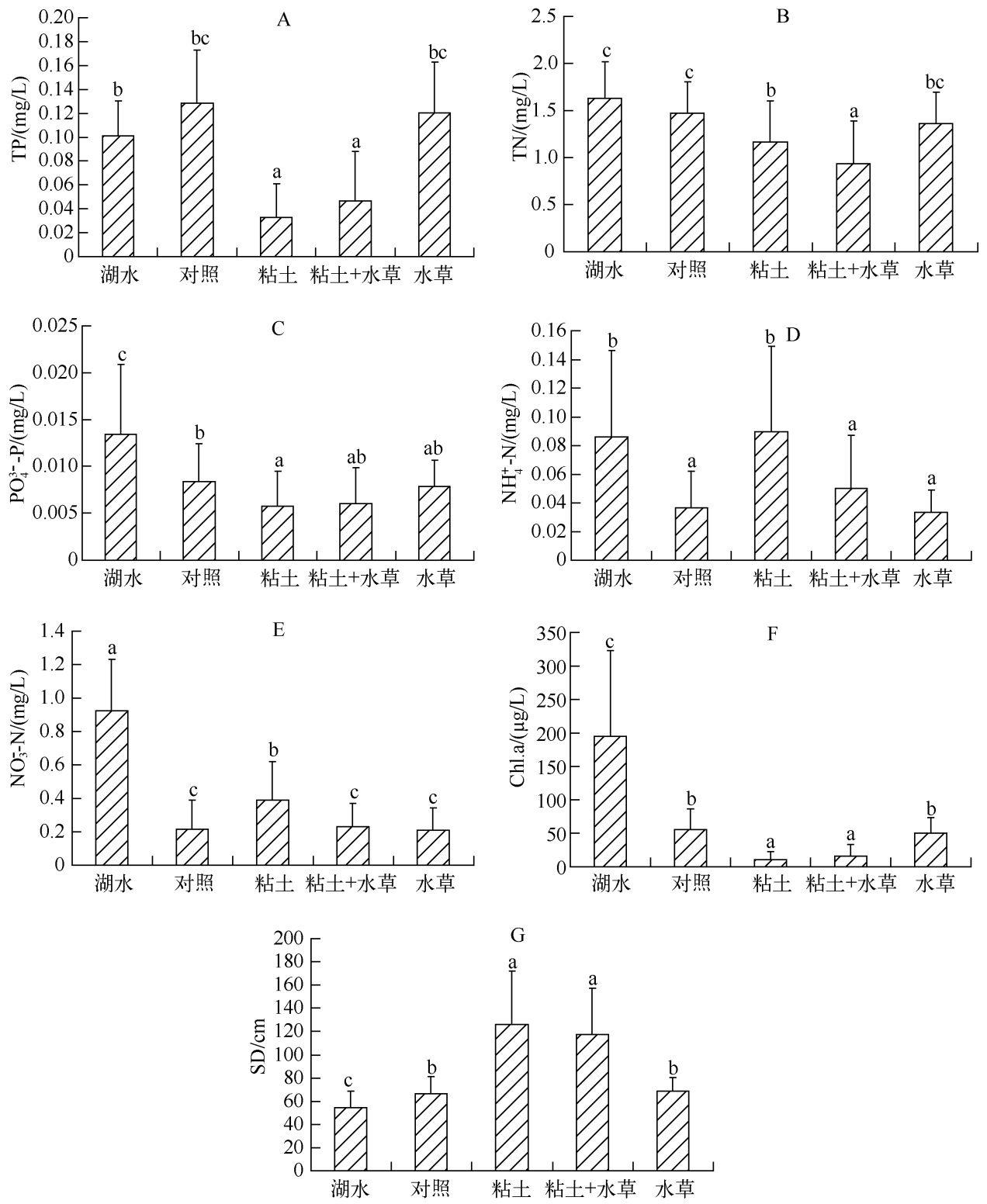

图1 四组处理围隔内水体和湖水的理化指标差异 (数据为平均值 $(n=57)$ 和湖水平均值 $(n=19)$, 误差线 代表标准差, 图中 a,b,c 字母表示 Tukey HSD 多重比较检验结果, 不同字母表示差异显著 $(P<0.05))$

Fig. 1 Comparison of the physicochemical indices in the four enclosure treatments and lake water

水草 + 粘土围隔底泥间隙水 TN 实验前为 $16 \mathrm{mg} / \mathrm{L}$, 实验后降到 $13 \mathrm{mg} / \mathrm{L}$; 水草围隔底泥间隙水 $\mathrm{TN}$ 实验前为 $14 \mathrm{mg} / \mathrm{L}$, 实验后升高到 $15 \mathrm{mg} / \mathrm{L}$, 粘土和水草 + 粘土围隔使间隙水 TN 相比实验前降低约 $15 \%$, 改性粘土和 移栽苦草处理对间隙水 $\mathrm{TN}$ 影响不显著 (图 $2 \mathrm{~A}$ ). 对照和粘土围隔实验前 $\mathrm{NH}_{4}^{+}-\mathrm{N}$ 含量约为 $3.4 \mathrm{mg} / \mathrm{L}$, 实验后 下降为 $1.0 \mathrm{mg} / \mathrm{L}$, 降幅分别为 $67 \%$ 和 $70 \%$; 水草 + 粘土和水草围隔实验前 $\mathrm{NH}_{4}^{+}-\mathrm{N}$ 含量约为 $4.0 \mathrm{mg} / \mathrm{L}$, 实验 后分别降为 $0.7 \mathrm{mg} / \mathrm{L}$ 和 $0.5 \mathrm{mg} / \mathrm{L}$, 降幅达 $83 \%$ 和 $86 \%$, 改性粘土和移栽苦草处理对间隙水 $\mathrm{NH}_{4}^{+}-\mathrm{N}$ 影响不 显著 (图 2D). 对照和粘土围隔实验前 $\mathrm{NO}_{3}^{-}-\mathrm{N}$ 含量为 $0.5 \mathrm{mg} / \mathrm{L}$, 实验后对照围隔 $\mathrm{NO}_{3}^{-}-\mathrm{N}$ 降为 $0.2 \mathrm{mg} / \mathrm{L}$, 而 粘土围隔 $\mathrm{NO}_{3}^{-}-\mathrm{N}$ 上升到 $0.87 \mathrm{mg} / \mathrm{L}$, 涨幅达 $68 \%$; 水草 + 粘土围隔实验前后 $\mathrm{NO}_{3}^{-}-\mathrm{N}$ 含量基本保持在 $0.6 \mathrm{mg} / \mathrm{L}$, 而水草围隔 $\mathrm{NO}_{3}^{-}-\mathrm{N}$ 由实验前的 $0.34 \mathrm{mg} / \mathrm{L}$ 上升到了 $0.78 \mathrm{mg} / \mathrm{L}$, 涨幅达 $130 \%$, 改性粘土和移栽 
苦草处理对间隙水 $\mathrm{NO}_{3}^{-}-\mathrm{N}$ 影响显著 (图 $2 \mathrm{E}$ ), 两者交互作用十分显著 (表 $1, P<0.05$ ).

对照围隔间隙水 $\mathrm{TP}$ 由实验前的 $0.6 \mathrm{mg} / \mathrm{L}$ 升高到实验后的 $1.2 \mathrm{mg} / \mathrm{L}$; 粘土围隔 $\mathrm{TP}$ 由实验前 $0.35 \mathrm{mg} / \mathrm{L}$ 降至 $0.20 \mathrm{mg} / \mathrm{L}$, 降幅达 40\% ; 水草 + 粘土围隔 TP 由实验前的 $0.3 \mathrm{mg} / \mathrm{L}$ 降至 $0.1 \mathrm{mg} / \mathrm{L}$, 降幅达 $60 \%$; 水草 围隔 TP 由实验前的 $0.3 \mathrm{mg} / \mathrm{L}$ 升高到 $0.4 \mathrm{mg} / \mathrm{L}$, 改性粘土和移栽苦草处理对间隙水 TP 影响显著(图 $2 B$ ). 对照围隔间隙水 $\mathrm{PO}_{4}^{3-}-\mathrm{P}$ 由实验前的 $0.55 \mathrm{mg} / \mathrm{L}$ 升高到实验后的 $0.70 \mathrm{mg} / \mathrm{L}$; 粘土围隔 $\mathrm{PO}_{4}^{3-}-\mathrm{P}$ 由实验前的 $0.35 \mathrm{mg} / \mathrm{L}$ 降低至实验后的 $0.08 \mathrm{mg} / \mathrm{L}$, 降幅达 $75 \%$; 水草 + 粘土围隔 $\mathrm{PO}_{4}^{3-}-\mathrm{P}$ 由实验前的 $0.14 \mathrm{mg} / \mathrm{L}$ 降低至 实验后的 $0.06 \mathrm{mg} / \mathrm{L}$, 降幅达 $55 \%$; 水草围隔 $\mathrm{PO}_{4}^{3-}-\mathrm{P}$ 由实验前的 $0.1 \mathrm{mg} / \mathrm{L}$ 升高到 $0.3 \mathrm{mg} / \mathrm{L}$, 改性粘土和移 栽苦草处理对间隙水 $\mathrm{PO}_{4}^{3-}-\mathrm{P}$ 影响显著 (图 2C), 两者也有显著交互作用 (表 $1, P<0.05$ ). 综上所述, 水草 + 粘土处理改善底质效果最好, 使间隙水的 $\mathrm{TN} 、 \mathrm{TP} 、 \mathrm{PO}_{4}^{3-}-\mathrm{P} 、 \mathrm{NH}_{4}^{+}-\mathrm{N}$ 分别比实验前下降了 $15.6 \% 、 61.7 \%$ 、 $55.8 \%$ 和 $82.8 \%, \mathrm{NO}_{3}^{-}-\mathrm{N}$ 含量基本不变.
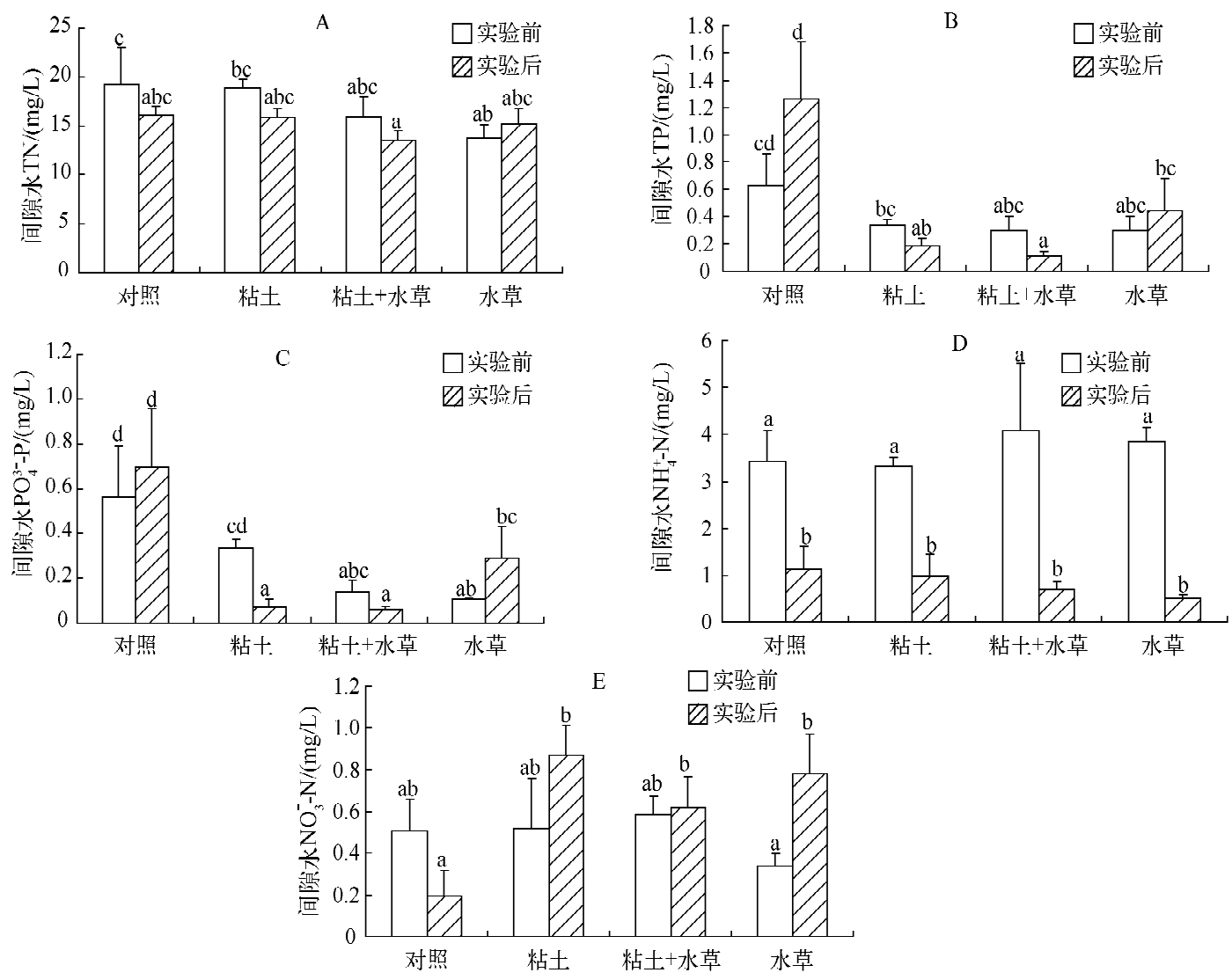

图 2 试验前后四组处理围隔内间隙水水化指标变化 (数据为平均值 $(n=3)$, 误差线代表标准差, 图中 $\mathrm{a}, \mathrm{b}, \mathrm{c}, \mathrm{d}$ 字母表示对实验后数据的 Tukey HSD 多重比较检验结果, 不同字母表示差异显著 $(P<0.05))$

Fig. 2 Variations of the interstitial water chemistry indices in the four enclosure treatments before and after the experiment

\section{3 苦草盖度对比和后期观察结果}

实验结束时水草 + 粘土围隔苦草盖度约 50\%, 水草围隔苦草盖度约 13\%, $t$ 检验显示这两组处理的植 被盖度有极显著差异 $(P<0.001)$. 实验结束后 3 个月定期观察发现, 水草 + 粘土围隔的透明度在 $70 \mathrm{~cm}$, 苦 草到 10 月份仍有大量存活, 其它处理组围隔的透明度约 $30 \mathrm{~cm}$, 水草围隔在 8 月中旬因蓝藻水华暴发导致 苦草地上部分几近全部死亡. 


\section{3 讨论}

本研究中, 单独改性粘土处理可以有效改善围隔水体中的 $\mathrm{TN} 、 \mathrm{TP} 、 \mathrm{PO}_{4}^{3-}-\mathrm{P} 、 \mathrm{Chl} . \mathrm{a}$ 和 $\mathrm{SD}$ 等指标, 但使 $\mathrm{NO}_{3}^{-}-\mathrm{N}$ 和 $\mathrm{NH}_{4}^{+}-\mathrm{N}$ 含量增加显著, 其对 $\mathrm{TN}$ 含量降低幅度显著小于水草 + 粘土处理, 后者相比粘土处理能显 著降低该两种离子态氮含量 $(P<0.05)$, 而水草单独处理对水体各指标影响不显著 (图 1). 底泥间隙水 TP、 $\mathrm{PO}_{4}^{3-}-\mathrm{P}$ 在粘土和苦草处理中降低显著, $\mathrm{NO}_{3}^{-}-\mathrm{N}$ 显著升高, 两种处理对 $\mathrm{PO}_{4}^{3-}-\mathrm{P}$ 和 $\mathrm{NO}_{3}^{-}-\mathrm{N}$ 含量交互作用非常 显著(表 1 和图 2,P<0.05), 但所有处理的间隙水 $\mathrm{TN} 、 \mathrm{NH}_{4}^{+}-\mathrm{N}$ 含量变化没有显著性差异. 粘土因其表面结 构的特殊性,对一些小分子物质具有吸附作用 ${ }^{[18]}$, 但对活性不高的 $\mathrm{NO}_{3}^{-}-\mathrm{N}$ 基本无吸附作用 ${ }^{[19]}$. 壳聚糖是一 种带正电荷的高分子聚凝物质, 它可以通过网桥作用絮凝一些小分子物质 ${ }^{[20]}$, 并且对小分子阳离子电解质 有排斥作用, 阳离子半径越小排斥越强 ${ }^{[21]}$, 因此本实验中改性粘土对 $\mathrm{NH}_{4}^{+}-\mathrm{N} \mathrm{NO}_{3}^{-}-\mathrm{N}$ 含量下降影响不显著. 经壳聚糖改性的粘土因为能加强网桥结构, 进而有效使藻类等悬浮颗粒物质絮凝沉降, 显著降低水体中的 $\mathrm{TN} 、 \mathrm{TP}$ 和 Chl. a 含量, 提高 $\mathrm{SD}^{[11-12]}$. 但是絮凝沉降到水底的藻类等会死亡分解, 即藻细胞膜破裂释放出大量 的胞内物质 ${ }^{[22]}$, 其中大量胶体和颗粒态有机氮经微生物降解会增大水体中的氮营养盐 ${ }^{[23]}$, 形成二次污染. 这可能是造成改性粘土处理的围隔水体中 $\mathrm{NO}_{3}^{-}-\mathrm{N} 、 \mathrm{NH}_{4}^{+}-\mathrm{N}$ 含量和间隙水中 $\mathrm{NO}_{3}^{-}-\mathrm{N}$ 含量显著增加的原因. 而改性高岭石粘土既能通过粘土中的铝层与 $\mathrm{PO}_{4}^{3-}-\mathrm{P}$ 反应 ${ }^{[19]}$, 也能通过壳聚糖分子上大量的氨基阳离子凝 聚带负电的藻细胞等 ${ }^{[10]}$, 即与 $\mathrm{PO}_{4}^{3-}-\mathrm{P}$ 发生电荷中和反应, 达到强烈的吸附效果. 因此, 这种改性粘土对 $\mathrm{PO}_{4}^{3-}-\mathrm{P}$ 吸附能力显著,在本实验中粘土处理磷酸盐含量都是显著减小的.

沉水植物既可从根系吸收来自于底泥的营养物质, 也能通过茎叶吸收来自水体的营养物质 ${ }^{[25-27]}$. 而且 沉水植物所需的氮和磷几乎全部来自底泥 ${ }^{[25-26]}$, 相比 $\mathrm{NO}_{3}^{-}-\mathrm{N}$ 对 $\mathrm{NH}_{4}^{+}-\mathrm{N}$ 更具有吸收优先性, 其代谢活动还 能够使底泥中硝酸盐的浓度显著提高, 原因可能是水生植物通过根系向底泥输送氧气, 提高了底质的氧化 还原电位, 从而有利于硝化作用 ${ }^{[27]}$. 沉水植物还能抑制底泥的再悬浮从而减少底泥向上覆水中营养盐的释 放 ${ }^{[4]}$. 因此苦草处理对底泥间隙水中磷元素和 $\mathrm{NO}_{3}^{-}-\mathrm{N}$ 影响显著, 能缓解粘土处理中 $\mathrm{NO}_{3}^{-}-\mathrm{N}$ 和 $\mathrm{NH}_{4}^{+}-\mathrm{N}$ 含量 升高的趋势. 与张木兰等 ${ }^{[24]}$ 的短期室内水质变化试验相比, 本研究反映了野外条件下壳聚糖改性粘土处理 富营养水体对水质变化的长期变化趋势,即对水体营养盐还有“升氮降磷”的效果.

富营养水体中较高的叶绿素藻类含量会导致沉水植物的衰退 ${ }^{[28]}$, 同时弱光照和高营养对沉水植物也造 成生理胁迫 ${ }^{[29-30]}$, 降低了沉水植物对浮游植物的竞争能力. Cao 等研究发现水体中 $0.3 \mathrm{mg} / \mathrm{L}$ 铵态氮可对苦 草造成生理胁迫 ${ }^{[31]}$. 本实验期间围隔水体的 $\mathrm{NH}_{4}^{+}-\mathrm{N}$ 含量仅为 $0.05 \mathrm{mg} / \mathrm{L}$, 远小于胁迫浓度, Zhang 等也发现 除了水华衰亡的时期, 在水生植物生长季节太湖蓝藻暴发严重的梅梁湾中铵态氮浓度一般很低, 这是铵态 氮被浮游植物优先吸收的结果, 而蓝藻暴发引起的低光低氧和产生的高浓度微囊藻毒素对水生植物的胁迫 在水华期非常显著 ${ }^{[32]}$. 陈开宁等 ${ }^{[33]}$ 在滇池进行的大型围隔实验也证明蓝藻能显著抑制竹叶眼子菜 (Potamogeton malaianus) 生长, 甚至使其大量死亡. 这可能是因为单独水草处理死亡率高, 难以度过水华胁迫期. 相反, 壳聚糖改性粘土因能加强网桥结构, 进而有效使藻类等悬浮颗粒物质絮凝沉降, 显著降低水体营养盐 和叶绿素含量, 改善透明度和光照强度,使沉水植物能正常生长并度过水华期. Pan 等 ${ }^{[11]}$ 在太湖运用该改性 粘土进行了沉水植被恢复和相对简单的水质监测实验, 但未说明恢复的具体长期效果, 本研究阐明了水 草 + 粘土处理蓝藻水华对水质的长期生态效应和原位修复本地沉水植被的长期监测结果.

本研究结果表明辅助壳聚糖改性粘土处理对围隔水体理化性质具有快速明显改善作用,使 TN、TP 和叶 绿素含量等大幅降低, 透明度增加 $50 \mathrm{~cm}$ 以上. 实验中水草 + 粘土相结合的方法使沉水植物修复显著成功, 苦草盖度远大于直接移栽水草的围隔, 并且在粘土处理的帮助下, 该围隔内苦草群落可以度过水华胁迫期, 并体现出较好水质改善效果. 相反在藻类密度高的围隔中直接移栽的苦草, 没有出现明显的生长和水质改 善效果. 因此本研究表明, 采用水草 + 粘土处理是改善富营养水体水质的有效途径, 而在低透明度下移栽的 沉水植物难以存活且对水质的改善效应不显著.

致谢: 感谢易春龙和罗从强等在采样和水质分析上的帮助.

\section{4 参考文献}

[ 1 ] 谢 平. 微囊藻毒素对人类健康影响相关研究的回顾. 湖泊科学,2009,21 (5):603-613. 
[ 2 ] Orth RJ, Moore KA. Chesapeake Bay: an unprecedented decline in submerged aquatic vegetation. Science, 1983,222 : 51-53.

[ 3 ] Moss B. Engineering and biological approaches to the restoration from eutrophication of shallow lakes in which aquatic plant communities are important components. Hydrobiologia, 1990, 200/201 (1) : 367-377.

[ 4 ] Scheffer M. Ecology of shallow lakes. New York: Chapman \& Hall, 1998: 20-234.

[ 5 ] 秦伯强, 高 光, 胡维平等. 浅水湖泊生态系统恢复的理论与实践思考. 湖泊科学, 2005,17(1):9-16.

[6]古滨河. 美国 Apopka 湖的富营养化及其生态恢复. 湖泊科学,2005,17(1):1-8.

[ 7 ] Qiu DG, Wu ZB, Liu BY et al. The restoration of aquatic macrophytes for improving water quality in a hypertrophic shallow lake in Hubei Province, China. Ecological Engineering, 2001, 18: 147-156.

[ 8 ] Morris K, Harrison KA, Bailey PCE et al. Domain shifts in the aquatic vegetation of shallow urban lakes: The relative roles of low light and anoxia in the catastrophic loss of the submerged angiosperm Vallisneria americana. Marine \& Freshwater Research, 2004, 55 : 749-758.

[ 9 ] Anderson DM. Turning back the harmful red tide. Nature, 1997, 388: 513-514.

[10］邹 华,潘 纲,陈 影. 壳聚糖改性粘土对水华优势藻铜绿微囊藻的絮凝去除. 环境科学, 2004,25 (6) :40-43.

[11] Pan G, Yang B, Wang D et al. In-lake algal bloom removal and submerged vegetation restoration using modified local soils. Ecological Engineering, 2011, 37 : 302-308.

[12] Zou H, Pan G, Chen H et al. Removal of cyanobacterial blooms in Lake Taihu using local soils. II. Effective removal of Microcystis aeruginosa using local soils and sediments modified by chitosan. Environmental Pollution, 2006, 141: 201-205.

[13] Pan G, Zou H, Chen H et al. Removal of harmful cyanobacterial blooms in Taihu Lake using local soils. III. Factors affecting the removal efficiency and an in situ field experiment using chitosan-modified local soils. Environmental Pollution, $2006,141(2)$ : 206-212.

[14] 胡志新, 胡维平, 张发兵等. 太湖梅梁湾生态系统健康状况周年变化的评价研究. 生态学杂志, 2005,24 (7): 364-368.

[15] Divakaran R, Pillai VNS. Flocculation of kaolinite suspensions in water by chitosan. Water Research, 2001, 35 (16): 3904-3908.

[16] 黄祥飞. 湖泊生态调查观测与分析. 北京:中国标准出版社,2000.

[17］国家环境保护总局. 水和废水监测分析方法:第 4 版. 北京:中国环境科学出版社,2002:88-438.

[18] Swartzen-Allen SL, Matijevic E. Surface and colloid chemistry of clays. Chemical Review, 1974, 74(3) : 385-400.

[19] 高咏卉,俞志明,宋秀贤等. 有机改性黏土对海水中营养盐及主要水质因子的影响. 海洋科学, 2007,31(8):30-37.

[20] Strand SP, Nordengen T, Hstgaard K. Efficiency of chitosans applied for flocculation of different bacteria. Water Research, 2002, 36: 4745-4752.

[21] 蒋文华,韩世钧. 粘度法研究壳聚糖对外加盐的敏感性. 高分子学报, 1998,5:567-572.

[22] Shao JH, Wang ZJ, Liu Y et al. Physiological responses of Microcystis aeruginosa NIES-843 (cyanobacterium) under the stress of chitosan modified kaolinite(CMK) loading. Ecotoxicology, 2012, 21(3) : 698-704.

[23] 孙小静,秦伯强, 朱广伟. 蓝藻死亡分解过程中胶体态磷、氮、有机碳的释放. 中国环境科学, 2007,27(3):341-345.

[24] 张木兰,潘 纲,陈 影等. 改性沉积物除藻对水质改善的效果研究.环境科学学报,2007,27 (1):13-17.

[25] Carignan R, Kalff J. Phosphorus sources for aquatic weeds: water or sediments? Science, 1980, 207: 987-989.

[26] Carr GM, Chambers PA. Macrophyte growth and sediment phosphorus and nitrogen in a Canadian prairie river. Freshwater Biolology, 1998, 39: 525-536.

[27] Barko JW, James WF. Effects of submerged aquatic macrophytes on nutrient dynamics, sedimentation and resuspension. In: Jeppesen E, Sondergaard M, Christoffersen K eds. The structuring role of submerged macrophytes in lakes. New York: Springer-Verlag, 1998: 197-214.

[28] Short FT, Burdick DM, Kaldy JEK. Mesocosm experiments quantify the effects of eutrophication on eelgrass, Zostera marina. Limnol \& Oceanogr, 1995, 40: 740-749.

[29] Ni LY. Growth of potamogeton maackianus under low-light stress in eutrophic water. Freshw Ecol, 2001, 16: $249-256$.

[30］黎慧娟,倪乐意, 曹 特等. 弱光照和富营养对苦草生长的影响. 水生生物学报,2008,32(2):225-230.

[31] Cao T, Xie P, Ni LY et al. The role of $\mathrm{NH}_{4}$ toxicity in the decline of the submersed macrophyte Vallisneria natans in lakes of the Yangtze River basin, China. Mar Freshwater Res, 2007, 58 : 581-587.

[32] Zhang M, Wang ZQ, Xu J et al. Ammonium, microcystins, and hypoxia of blooms in eutrophic water cause oxidative stress and C-N imbalance in submersed and floating-leaved aquatic plants in Lake Taihu, China. Chemosphere, 2011, 82 : 329-339.

[33] 陈开宁,李文朝, 吴庆龙等. 滇池蓝藻对沉水植物生长的影响. 湖泊科学, 2003,15(4) :364-368. 